\title{
Prognostic significance of CDX2 and mucin expression in small intestinal adenocarcinoma
}

\author{
Sun-Young Jun ${ }^{1,6}$, Dae Woon Eom ${ }^{2,6}$, Hosub Park ${ }^{3}$, Young Kyung Bae ${ }^{4}$, Kee-Taek Jang ${ }^{5}$, \\ Eunsil $\mathrm{Yu}^{3}$ and Seung-Mo Hong ${ }^{3}$ \\ ${ }^{1}$ Department of Pathology, Incheon St. Mary's Hospital, The Catholic University of Korea, Incheon, Republic \\ of Korea; ${ }^{2}$ Department of Pathology, Gangneung Asan Hospital, University of Ulsan College of Medicine, \\ Gangneung, Republic of Korea; ${ }^{3}$ Department of Pathology, Asan Medical Center, University of Ulsan College \\ of Medicine, Seoul, Republic of Korea; ${ }^{4}$ Department of Pathology, Yeungnam University College of Medicine, \\ Daegu, Republic of Korea and ${ }^{5}$ Department of Pathology, Samsung Medical Center, Sungkyunkwan \\ University School of Medicine, Seoul, Republic of Korea
}

\begin{abstract}
The clinicopathological and prognostic significance of CDX2 and mucin expression have not been comprehensively evaluated in small intestinal adenocarcinoma. Immunohistochemical microarray analyses of CDX2, MUC1, MUC5AC, and MUC6 protein expressions in 189 surgically resected small intestinal adenocarcinoma cases were examined and compared with various clinicopathologic variables, including survival. CDX2, MUC1, MUC5AC, and MUC6 expressions were observed in $43.4 \%$ (82 patients), $37.6 \%$ (71), $31.7 \%$ (60), and $21.7 \%$ (41) of patients, respectively. Whereas CDX2 expression was found to be associated with lowgrade tumors $(P=0.034)$, fewer nodal metastases $(P=0.019)$, and less perineural invasion $(P=0.049)$ in small intestinal adenocarcinoma patients, patients expressing MUC1 tended to demonstrate high-grade $(P=0.021)$ and nodular or infiltrative $(P=0.020)$ tumors. On the basis of the combined CDX2, MUC1, MUC5AC, and MUC6 expression patterns, small intestinal adenocarcinoma patients were further classified as intestinal (CDX2 + /MUC1 - ; 29.6\%), pancreatobiliary (CDX2 - /MUC1 + ; 23.8\%), mixed (CDX2 + /MUC1 + ; 13.8\%), gastric (CDX2 - /MUC1 - /MUC5AC + or MUC6 +; 13.8\%), or null (CDX2 - /MUC1 - /MUC5AC - /MUC6 - ; 19.0\%). Among these immunophenotypes, intestinal-type patients demonstrated more frequent distal (jejunal or ileal; $P=0.033)$, tubular $(P=0.039)$, and low-grade tumors $(P=0.004)$ and significantly better survival according to univariate $(P<0.0001)$ and multivariate $(P=0.001)$ analyses. In summary, intestinal immunophenotype adenocarcinomas are associated with distal (jejunal or ileal), tubular, and low-grade tumors and better survival outcomes. Hence, CDX2 and mucin immunohistochemical staining may provide better estimations of survival after surgical resection and intestinal immunophenotype could therefore be used as a better prognostic indicator of small intestinal adenocarcinoma.
\end{abstract}

Modern Pathology (2014) 27, 1364-1374; doi:10.1038/modpathol.2014.36; published online 7 March 2014

Keywords: adenocarcinoma; CDX2; immunohistochemistry; mucin; prognosis; small intestine

Primary small intestinal adenocarcinomas account for only $5 \%$ of malignant neoplasms in the gastrointestinal tract despite the long length of the small intestine and the significant mucosal coverage over the entire gastrointestinal tract. ${ }^{1}$ In 2013 , it is estimated that 8810 Americans will be diagnosed

Correspondence: Professor S-M Hong, MD, PhD, Department of Pathology, Asan Medical Center, University of Ulsan College of Medicine, 88, Olympic-ro 43-gil, Songpa-gu, Seoul 138-736, Republic of Korea.

E-mail: smhong28@gmail.com

${ }^{6}$ These authors contributed equally to this work.

Received 25 July 2013; revised 30 December 2013; accepted 31

December 2013; published online 7 March 2014 with small intestinal adenocarcinoma. ${ }^{1}$ Despite recent improvements in the detection of the small intestinal adenocarcinomas, including improved imaging techniques and endoscopic modalities, the diagnosis of small intestinal adenocarcinomas is usually made at an advanced clinical stage and the 5-year survival rate is only $41.2 \% .^{2}$ Several clinicopathologic factors, including lymph node metastasis and the distal locations of these tumors (jejunum and ileum), are known as the most important independent prognostic factors. ${ }^{2}$ Although several molecular alterations, including $K R A S$, TP53, and DPC4/SMAD4 mutations and the overexpression of cyclinD1, are known to be involved in small intestinal adenocarcinoma carcinogenesis, ${ }^{3-6}$ 
only a few of these, such as $\beta$-catenin and e-cadherin, demonstrate prognostic implications for small intestinal adenocarcinoma patients. ${ }^{7}$

Mucins are large filamentous glycoproteins that heavily glycosylate many of the oligosaccharide side chains that are linked to the protein backbone called apomucin. ${ }^{8}$ To date, $\geq 19$ mucins have been identified and divided into two distinct classes according to their structure and function: (1) membrane-associated types (MUC-1, -3A, -3B, -4, $-12,-13,-15,-16,-17$, and -20); and (2) secreted types (MUC-2, -5 AC, $-5 B,-6,-7,-8,-9$, and -19). ${ }^{9}$ Membrane-associated mucins are involved in epithelial cell-cell interactions, whereas secreted mucins comprise the major macromolecular component of mucus. ${ }^{10}$ The expression patterns appear to be relatively cell, tissue, and organ specific, especially those of secreted mucins. ${ }^{9}$ Among mucin proteins, MUC1, MUC5AC, and MUC6 have been investigated in various gastrointestinal organs. ${ }^{11}$ In the normal mucosa of the gastrointestinal tract, MUC1 (also known as epithelial membrane antigen), which is located on chromosome 7q22, is mainly expressed in the pancreatic ducts and superficial foveolar epithelium. ${ }^{12}$ MUC5AC and MUC6, which are located on chromosome 11p15, demonstrate considerably similar overall homologies. ${ }^{12}$ MUC5AC is predominantly expressed in gastric foveolar epithelial cells and is considered to be a major gastric mucin. MUC6 is also expressed in the gastric pyloric glands. Mucin proteins have also been extensively studied to determine their clinical and prognostic significance in gastrointestinal tract cancers. ${ }^{13-19}$ A previous study reported that MUC5AC and MUC6 coexpression is associated with better survival in patients with ampullary adenocarcinomas, especially among patients with tumors expressing or lacking both CK20 and MUC1. ${ }^{13}$ Several other studies reported that MUC1 expression is associated with worse survival in stomach cancer patients compared with patients without MUC1 expression. ${ }^{16,17,19}$ However, no previous study has compared the overall mucin expression status and its clinical and prognostic significance in small intestinal adenocarcinoma patients. Hence, further studies are needed to evaluate the implications of the mucin expression status in small intestinal adenocarcinomas.

CDX2 is a Drosophila caudal-related homeobox transcription factor responsible for early intestinal differentiation. ${ }^{20} \mathrm{CDX} 2$ is normally present throughout embryonic and postnatal life within the nuclei of the intestinal epithelial cells in the proximal duodenum through the distal rectum. ${ }^{21}$ CDX2 stimulates intestinal proliferation and differentiation through the transcriptional activation of intestine-specific proteins, including MUC2. ${ }^{22}$ Several previous reports have suggested a tumorsuppressive role for CDX2 in human colorectal and gastric carcinogenesis. ${ }^{23} \mathrm{CDX} 2$ expression is associated with not only intestinal mucin phenotypes, ${ }^{14,24}$ but also an early tumor stage ${ }^{24-30}$ and improved prognosis in patients with gastric cancer. ${ }^{29-32}$ A previous study reported that CDX2 expression is also correlated with an improved prognosis in patients with ampullary adenocarcinomas. ${ }^{33}$

Several previous studies have compared the protein expression profiles of gastric and colonic carcinomas and small intestinal adenocarcinomas because of the anatomical proximity and similarities between these cancers. Although a few studies have reported the expression patterns of CDX2 and mucin proteins in small intestinal adenocarcinomas and ampullary carcinomas, most of these analyses were performed on a small number of small intestinal adenocarcinoma patients. ${ }^{11,13,21,33-38}$ Moreover, to the best of our knowledge, no previous studies have investigated the prognostic significance of CDX2 and mucin expression in small intestinal adenocarcinomas.

In our present study, we analyze the expression of CDX2, MUC1, MUC5AC, and MUC6 proteins in small intestinal adenocarcinomas and evaluate the immunophenotypic subtypes of these lesions in accordance with the combined expression patterns of these proteins. We further evaluate any correlations between clinicopathologic and prognostic significance, the combined expression patterns of CDX2 and mucins, and the defined immunophenotypes of the small intestinal adenocarcinomas.

\section{Materials and methods}

\section{Tissue Samples}

After receiving approvals from the institutional review boards of each participating institution, a total cohort of 197 surgically resected primary small intestinal adenocarcinomas were collected from the surgical pathology archives of 22 South Korean institutions by the Korean Small Intestinal Cancer Study Group, as previously reported. ${ }^{2}$ Carcinomas originating from the mucosa of the small intestines, including the duodenum, jejunum, and ileum, were included in our present study. Carcinomas extending from the surrounding gastrointestinal tract organs, such as the stomach, ampulla of Vater, pancreas, cecum, or appendix, into the small intestines were excluded from the analysis.

Clinical and pathologic data that were collected as part of our previous study were used again in our current study. ${ }^{2}$ Clinical data included patient sex, age, tumor location, operation date, TNM stage, most recent follow-up examination, survival status, presence of synchronous or metachronous malignancies, and presence or absence of predisposing conditions of small intestinal adenocarcinomas, including Crohn's disease, familial adenomatous polyposis, Lynch syndrome, Peutz-Jeghers syndrome, Gardner's syndrome, gluten-sensitive enteropathy, intestinal duplication, Meckel's diverticulum, or 
ectopic pancreas. Pathological data obtained from the gross examination included tumor size and growth pattern. Macroscopic growth pattern of small intestinal adenocarcinomas were divided into three groups, including polypoid pattern, exophytic with predominantly intraluminal growth; nodular pattern, endophytic/ulcerative with intramural growth; and infiltrative pattern, annular with circumferential involvement or diffusely infiltrative. ${ }^{39}$ The microscopic characteristics included histologic subtype, tumor grade, depth of invasion, peritoneal seeding, pancreatic and other intestinal loop invasions, nodal metastasis, and perineural and lymphovascular invasion.

Histologic types were classified according to the fouth edition of the WHO classification. ${ }^{39}$ Tumor grading was classified as low-grade (well ( $>95 \%$ with gland formation) and moderately differentiated (50-95\% with gland formation) adenocarcinomas) and high-grade (poorly differentiated (1-49\% with gland formation) and undifferentiated (no gland formation)) carcinomas following criteria for histological grading of colorectal adenocarcinoma, which was described in 2010 WHO classification. ${ }^{39}$

\section{Tissue Microarray}

Tissue microarrays were constructed from the archived formalin-fixed and paraffin-embedded tissue blocks, as previously described. ${ }^{7,40}$ Briefly, areas with invasive adenocarcinomas were identified on the corresponding hematoxylin and eosin-stained slides, and sections were indicated as a representative tumor or as normal small intestinal mucosa. Three cores from each tumor and 1 matched core from the normal small intestinal mucosal samples were sampled using a 1.0-mm punch. Tissue microarrays were constructed using a manual tissue arrayer (Beecher Instruments, Sun Prairie, WI, USA). Four$\mu \mathrm{m}$-thick slides were cut from the tissue microarray blocks for immunohistochemical staining.

\section{Immunohistochemistry}

Immunohistochemical staining was performed using primary mouse monoclonal antibodies (Table 1). Staining was conducted on a Ventana BenchMark XT automated slide stainer (Ventana Medical Systems, Tucson, AZ, USA) using a highpH CC1 buffer and the heat-induced epitope retrieval method. Staining was visualized using the ultraView DAB Universal Detection Kit (Ventana), which used a hydrogen peroxide substrate and 3,3'-diaminobenzidine chromogen solution. The slides were subsequently counterstained with hematoxylin. Immunoreactivity was interpreted by light microscopic examination and independently evaluated by two pathologists who authored this study (S-YJ and S-MH) and who were blind to the clinicopathological information. CDX2 was found to
Table 1 Antibodies used in this study

\begin{tabular}{lccl}
\hline Antibody & Clone & Dilution & Supplier \\
\hline CDX2 & Cdx2-88 & $1: 200$ & BioGenex \\
MUC1 & Ma695 & Prediluted & Novocastra \\
MUC5AC & CLH2 & $1: 200$ & Novocastra \\
MUC6 & CLH5 & $1: 200$ & Novocastra \\
\hline
\end{tabular}

be expressed in the nuclei of the tumor cells (Figure 1a), and MUC1 was expressed in a cytoplasmic pattern with membranous accentuation (Figure 1b). MUC5AC and MUC6 were both found to be expressed in the cytoplasm (Figure 1c and d). Immunostained slides were scored in accordance with the percentage of unequivocally positive epithelial cells as follows: 0 , staining in $\leq 5 \%$ of tumor cells; $1+$, staining in $6-25 \%$ of tumor cells; $2+$, staining in $26-50 \%$ of tumor cells; $3+$, staining in $51-75 \%$ of tumor cells; and $4+$, staining in $\geq 76 \%$ of tumor cells. Immunostaining scores were independently evaluated in each tumor core. Only sections demonstrating $>5 \%$ positively stained tumor cells were considered positive, as previously described. ${ }^{16,24,28,30,31,36}$ We selected CDX2-immunostained slides and evaluated 189 cases for immunopositivity, the $\kappa$ value for interobserver agreement of two pathologists (S-YJ and $\mathrm{S}-\mathrm{MH}$ ) was 0.85 , which indicated almost perfect agreement. Discrepancies were resolved by simultaneous reevaluation and discussion.

\section{Immunophenotypic Classification of Small Intestinal Adenocarcinomas}

We classified small intestinal adenocarcinomas according to their combined CDX2 and mucin immunophenotypes (Figure 2). On the basis of the combined CDX2 and MUC1 expression patterns, cases were classified as intestinal (CDX2 + MUC1 -), pancreatobiliary (CDX2 - /MUC1 +), or mixed (CDX2 +/MUC1 +). Within the CDX2-/ MUC1 - group, small intestinal adenocarcinomas exhibiting either MUC5AC + or MUC6 + immunoreactivity were categorized as gastric. The remaining type (CDX2 - /MUC1 - /MUC5AC - /MUC6 -) was classified as null.

\section{Statistical Analysis}

Statistical analyses were performed using SPSS software (version 17.0; SPSS, Chicago, IL, USA). Means were compared using the unpaired student $t$-test. The $\chi^{2}$ and Fisher exact tests were used to examine associations between categorical variables. Overall patient survival was defined as the time from surgical resection to death or the last follow-up examination. The survival rate was calculated using the Kaplan-Meier method. Associations between 

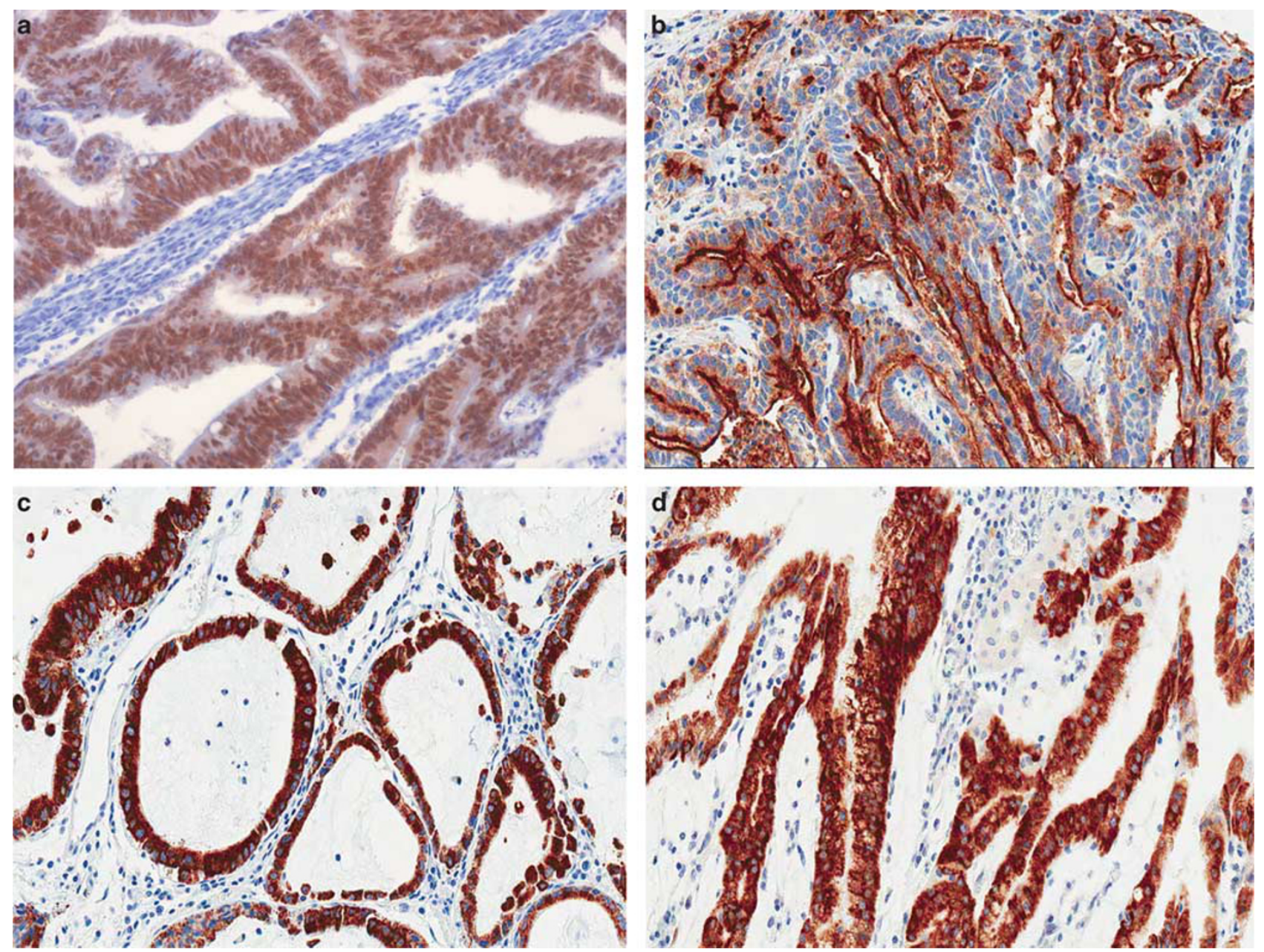

Figure 1 Expression profile of CDX2 and various mucins. (a) CDX2 is expressed in the nuclei of tumor cells; (b) MUC1 is expressed in a cytoplasmic pattern with membranous accentuation; and (c) MUC5AC and (d) MUC6 are expressed in the cytoplasm.

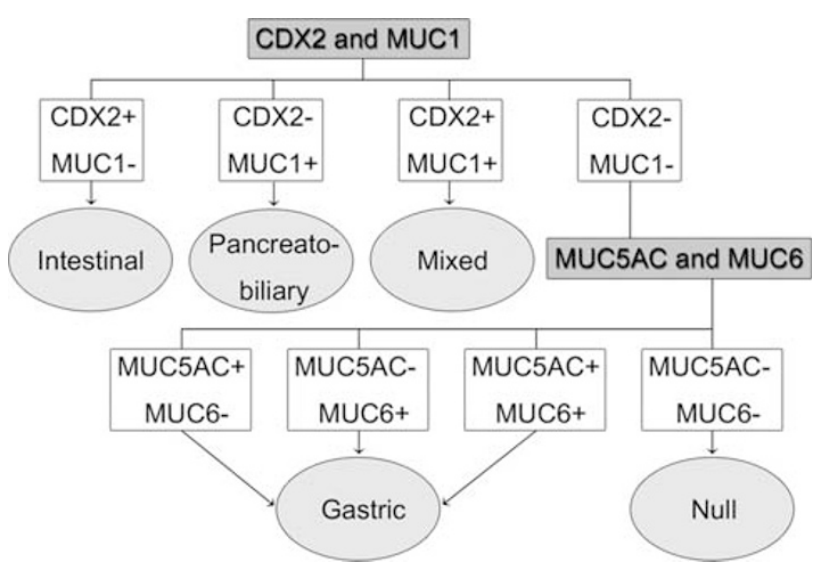

Figure 2 Immunophenotypic classification of small intestinal adenocarcinomas according to the expression patterns of CDX2 and various mucins. Small intestinal adenocarcinomas were classified as intestinal (CDX2 +/MUC1 - ), pancreatobiliary (CDX2 - /MUC1 +), mixed (CDX2 + /MUC1 +), or null (CDX2 - / MUC1 - /MUC5AC - /MUC6 - ). Within the CDX2 - /MUC1 group, cases exhibiting either MUC5AC or MUC6 immunopositivity were classified as gastric. survival rates and various clinicopathologic factors were assessed using the log-rank test. We also investigated the significance of any prognostic factors using Cox proportional hazards modeling. In this study, $P$ values $<0.05$ were considered to denote statistical significance.

\section{Results}

\section{Patient Characteristics}

Out of a total of 197 patients, 189 patients with interpretable immunohistochemical CDX2 and mucin expression results were included in our study cohort. Eight cases were not successfully immunostained in our subsequent analysis due to tissue loss during sectioning or to the staining process. Patient ages ranged between 23 and 86 years (mean, 59.0 years; standard deviation (s.d.), 14.0 years). The male:female ratio was 1.7. The follow-up period after surgical resection ranged 
between 0.3 and 158.2 months (mean, 37.1 months; median, 28.1 months). Several predisposing conditions were observed in 23 cases $(12.2 \%)$ ), including 17 cases with sporadic adenomas, 3 cases of PeutzJeghers syndrome, 2 cases with Meckel's diverticulum, and 1 case with Crohn's disease. On the basis of clinical definition of revised Bethesda criteria, ${ }^{41}$ there were 13 patients of suspected Lynch syndrome who had metachronous colorectal cancers. There was no patient with familial adenomatous polyposis, Gardner syndrome, gluten-sensitive enteropathy, intestinal duplication, and ectopic pancreas.

\section{Expression Levels of CDX2 and Mucin}

CDX2, MUC1, MUC5AC, and MUC6 expression levels were observed in $43.4 \%$ (82 patients), $37.6 \%$ (71), $31.7 \%$ (60), and $21.7 \%$ (41) of patients, respectively. CDX2 expression in small intestinal adenocarcinomas was found to be associated with low-grade tumors $(P=0.034)$, less nodal metastasis $(P=0.019)$, and less perineural invasion $(P=0.049)$. Patients with positive MUC1 expression tended to demonstrate high-grade tumors $(P=0.021)$ and nodular or infiltrative tumors $(P=0.020)$. MUC5AC + and MUC6 + adenocarcinomas frequently developed in the proximal (duodenal) location $(P=0.001$ and $P<0.0001$, respectively) and tended to demonstrate pancreatic invasion ( $P=0.047$ and $P=0.035$, respectively). MUC5AC + adenocarcinomas were typically low-grade tumors $(P=0.010)$. MUC6 + adenocarcinoma patients were predominantly male $(P=0.020)$, tended to develop tumors with a low $\mathrm{T}$ classification $(P=0.015)$, and presented less frequently with lymphovascular invasion ( $P=0.005$; Table 2$)$.

Tumor cells were positive for CDX2 and negative for all mucin proteins in one case with Crohn's disease. In the small intestinal adenocarcinomas of suspected Lynch syndrome patients, tumor cells were frequently negative for MUC1 (8/13 cases), MUC5A (12/13), and MUC6 (11/13) and positive for CDX2 (7/13) (Table 2). CDX2, MUC1, MUC5AC, and MUC6 expressions were not significantly different according to the histologic subtypes of small intestinal adenocarcinomas (Table 2). Five micropapillary carcinoma cases contained various degrees (20-60\% of total tumor volume) of micropapillary carcinoma component, which were not correlated with CDX2 or mucin expression (Table 2).

\section{Immunophenotypic Classification}

The immunophenotypic classifications and clinicopathologic factors of the small intestinal adenocarcinoma cohort are summarized in Table 3. On the basis of the combined expression patterns of CDX2 and mucins, the small intestinal adenocarcinomas in our current study were classified as intestinal (56
Table 2 Correlation between clinicopathological factors and CDX2, MUC1, MUC5AC, and MUC6 expression in the small intestinal adenocarcinoma study cohort

\begin{tabular}{|c|c|c|c|c|c|}
\hline \multirow[b]{2}{*}{ Clinicopathologic factors } & \multirow[b]{2}{*}{$\mathrm{n}$} & \multicolumn{4}{|c|}{ No. of positive patients } \\
\hline & & $C D X 2$ & MUC1 & $M U C 5 A C$ & MUC6 \\
\hline \multicolumn{6}{|l|}{ Sex } \\
\hline Male & 118 & $51(43 \%)$ & $50(42 \%)$ & $39(33 \%)$ & $32(27 \%)$ \\
\hline Female & 71 & $31(44 \%)$ & $21(30 \%)$ & $21(30 \%)$ & $9(13 \%)$ \\
\hline$P$ & & 0.953 & 0.079 & 0.619 & $0.020^{\mathrm{a}}$ \\
\hline \multicolumn{6}{|l|}{ Growth pattern ${ }^{\mathrm{b}}$} \\
\hline Polypoid & 34 & $13(38 \%)$ & $6(18 \%)$ & $8(24 \%)$ & $9(27 \%)$ \\
\hline Nodular & 12 & $8(67 \%)$ & $6(50 \%)$ & $4(33 \%)$ & $2(17 \%)$ \\
\hline Infiltrative & 135 & $59(44 \%)$ & $56(42 \%)$ & $45(33 \%)$ & $26(19 \%)$ \\
\hline$P$ & & 0.228 & $0.020^{\mathrm{a}}$ & 0.539 & 0.614 \\
\hline \multicolumn{6}{|l|}{ Location } \\
\hline Proximal (duodenum) & 101 & $39(39 \%)$ & $42(42 \%)$ & $43(43 \%)$ & $33(33 \%)$ \\
\hline Distal (jejunum, ileum) & 88 & $43(49 \%)$ & $29(33 \%)$ & $17(19 \%)$ & $8(9 \%)$ \\
\hline$P$ & & 0.156 & 0.222 & $0.001^{\mathrm{a}}$ & $<0.0001^{\mathrm{a}}$ \\
\hline \multicolumn{6}{|l|}{ Histological subtype } \\
\hline Tubular & 169 & $75(44 \%)$ & $61(36 \%)$ & $57(34 \%)$ & $38(23 \%)$ \\
\hline Mucinous & 8 & $3(38 \%)$ & $2(25 \%)$ & $1(13 \%)$ & $2(25 \%)$ \\
\hline Micropapillary & 5 & $2(40 \%)$ & $3(60 \%)$ & $1(20 \%)$ & $1(20 \%)$ \\
\hline Signet ring cell & 3 & $2(67 \%)$ & $2(67 \%)$ & $1(33 \%)$ & 0 \\
\hline Undifferentiated & 4 & 0 & $3(75 \%)$ & 0 & 0 \\
\hline$P$ & & 0.463 & 0.245 & 0.529 & 0.948 \\
\hline \multicolumn{6}{|l|}{ Grade } \\
\hline Low & 145 & $69(48 \%)$ & $48(33 \%)$ & $53(37 \%)$ & $35(24 \%)$ \\
\hline High & 44 & $13(30 \%)$ & $23(52 \%)$ & $7(16 \%)$ & $6(14 \%)$ \\
\hline$P$ & & $0.034^{\mathrm{a}}$ & $0.021^{\mathrm{a}}$ & $0.010^{\mathrm{a}}$ & 0.139 \\
\hline \multicolumn{6}{|l|}{$p T$ classification ${ }^{\mathrm{c}}$} \\
\hline $\mathrm{pT} 1+\mathrm{pT} 2$ & 16 & $8(50 \%)$ & $5(31 \%)$ & $8(50 \%)$ & $8(50 \%)$ \\
\hline pT3 & 61 & $31(51 \%)$ & $19(31 \%)$ & $19(31 \%)$ & $9(15 \%)$ \\
\hline pT4 & 109 & $41(38 \%)$ & $47(43 \%)$ & $33(30 \%)$ & $24(22 \%)$ \\
\hline$P$ & & 0.209 & 0.255 & 0.281 & $0.015^{\mathrm{a}}$ \\
\hline \multicolumn{6}{|l|}{$p N$ classification ${ }^{\mathrm{b}}$} \\
\hline pNo & 83 & $45(54 \%)$ & $28(34 \%)$ & $29(35 \%)$ & $21(25 \%)$ \\
\hline pN1 & 88 & $32(36 \%)$ & $36(41 \%)$ & $29(33 \%)$ & $18(21 \%)$ \\
\hline$P$ & & $0.019^{\mathrm{a}}$ & 0.333 & 0.784 & 0.450 \\
\hline \multicolumn{6}{|l|}{ Pancreatic invasion } \\
\hline No & 123 & $58(47 \%)$ & $41(33 \%)$ & $33(27 \%)$ & $21(17 \%)$ \\
\hline Yes & 66 & $24(36 \%)$ & $30(46 \%)$ & $27(41 \%)$ & $20(30 \%)$ \\
\hline$P$ & & 0.154 & 0.101 & $0.047^{\mathrm{a}}$ & $0.035^{\mathrm{a}}$ \\
\hline \multicolumn{6}{|l|}{ Other loop invasion } \\
\hline No & 184 & $80(44 \%)$ & $68(37 \%)$ & $59(32 \%)$ & $40(22 \%)$ \\
\hline Yes & 5 & $2(40 \%)$ & $3(60 \%)$ & $1(20 \%)$ & $1(20 \%)$ \\
\hline$P$ & & 1.000 & 0.366 & 1.000 & 1.000 \\
\hline \multicolumn{6}{|l|}{ Retroperitoneal seeding } \\
\hline No & 175 & $76(43 \%)$ & $65(37 \%)$ & $56(32 \%)$ & $37(21 \%)$ \\
\hline Yes & 14 & $6(43 \%)$ & $6(43 \%)$ & $4(29 \%)$ & $4(29 \%)$ \\
\hline$P$ & & 0.967 & 0.671 & 1.000 & 0.508 \\
\hline \multicolumn{6}{|l|}{ Perineural invasion } \\
\hline No & 126 & $61(48 \%)$ & $43(34 \%)$ & $43(34 \%)$ & $31(25 \%)$ \\
\hline Yes & 63 & $21(33 \%)$ & $28(44 \%)$ & $17(27 \%)$ & $10(16 \%)$ \\
\hline$P$ & & $0.049^{\mathrm{a}}$ & 0.167 & 0.320 & 0.170 \\
\hline \multicolumn{6}{|l|}{ Lymphovascular invasion } \\
\hline No & 91 & $45(50 \%)$ & $30(33 \%)$ & $33(36 \%)$ & $28(31 \%)$ \\
\hline Yes & 98 & $37(38 \%)$ & $41(42 \%)$ & $27(28 \%)$ & $13(13 \%)$ \\
\hline$P$ & & 0.109 & 0.231 & 0.214 & $0.005^{\mathrm{a}}$ \\
\hline \multicolumn{6}{|c|}{ Suspected Lynch syndrome } \\
\hline No & 176 & $75(43 \%)$ & $66(38 \%)$ & $59(34 \%)$ & $39(22 \%)$ \\
\hline Yes & 13 & $7(54 \%)$ & $5(39 \%)$ & $1(8 \%)$ & $2(15 \%)$ \\
\hline$P$ & & 0.430 & 1.000 & 0.065 & 0.737 \\
\hline
\end{tabular}

${ }^{\text {a}}$ Statistically significant $(P<0.05)$.

${ }^{\mathrm{b}}$ Calculated using only patients with sufficient available data.

${ }^{\mathrm{C}}$ Excluded patients with pTis. 
Table 3 Correlation between immunophenotypic classification and clinicopathologic factors in the small intestinal adenocarcinoma study cohort

\begin{tabular}{|c|c|c|c|c|c|c|c|}
\hline \multirow[b]{2}{*}{ Clinicopathologic factors } & \multirow[b]{2}{*}{$\mathrm{n}$} & \multicolumn{5}{|c|}{ No. of patients } & \multirow[b]{2}{*}{$\mathrm{P}$-value } \\
\hline & & Intestinal & Pancreatobiliary & Gastric & Mixed & Null & \\
\hline Growth pattern ${ }^{\mathrm{a}}$ & & & & & & & 0.172 \\
\hline Polypoid & 34 & $13(38 \%)$ & $6(18 \%)$ & $5(15 \%)$ & 0 & $10(29 \%)$ & \\
\hline Nodular & 12 & $4(33 \%)$ & $2(17 \%)$ & $1(8 \%)$ & $4(33 \%)$ & $1(8 \%)$ & \\
\hline Infiltrative & 135 & $37(27 \%)$ & $34(25 \%)$ & $18(13 \%)$ & $22(16 \%)$ & $24(18 \%)$ & \\
\hline Location & & & & & & & $0.033^{\mathrm{b}}$ \\
\hline Proximal (duodenum) & 101 & $26(26 \%)$ & $29(29 \%)$ & $19(19 \%)$ & $13(13 \%)$ & $14(14 \%)$ & \\
\hline Distal (jejunum, ileum) & 88 & $30(34 \%)$ & $16(18 \%)$ & $7(8 \%)$ & $13(15 \%)$ & $22(25 \%)$ & \\
\hline Histological subtype & & & & & & & $0.039^{b}$ \\
\hline Tubular & 169 & $54(32 \%)$ & $40(24 \%)$ & $25(15 \%)$ & $21(12 \%)$ & $29(17 \%)$ & \\
\hline Mucinous & 8 & $1(13 \%)$ & 0 & $1(13 \%)$ & $2(25 \%)$ & $4(50 \%)$ & \\
\hline Micropapillary & 5 & $1(20 \%)$ & $2(40 \%)$ & 0 & $1(20 \%)$ & $1(20 \%)$ & \\
\hline Signet ring cell & 3 & 0 & 0 & 0 & $2(67 \%)$ & $1(33 \%)$ & \\
\hline Undifferentiated & 4 & 0 & $3(75 \%)$ & 0 & 0 & $1(25 \%)$ & \\
\hline Tumor grade & & & & & & & $0.004^{\mathrm{b}}$ \\
\hline Low & 145 & $51(35 \%)$ & $30(21 \%)$ & $23(16 \%)$ & $18(12 \%)$ & $23(16 \%)$ & \\
\hline High & 44 & $5(11 \%)$ & $15(34 \%)$ & $3(7 \%)$ & $8(18 \%)$ & $13(30 \%)$ & \\
\hline$p T^{c}$ & & & & & & & 0.211 \\
\hline $\mathrm{pT} 1+\mathrm{pT} 2$ & 16 & $7(44 \%)$ & $4(25 \%)$ & $4(25 \%)$ & $1(6 \%)$ & 0 & \\
\hline pT3 & 61 & $21(34 \%)$ & $9(15 \%)$ & $9(15 \%)$ & $10(16 \%)$ & $12(20 \%)$ & \\
\hline pT4 & 109 & $26(24 \%)$ & $32(29 \%)$ & $13(12 \%)$ & $15(14 \%)$ & $23(21 \%)$ & \\
\hline$p N^{a}$ & & & & & & & 0.141 \\
\hline pNo & 83 & $30(36 \%)$ & $13(16 \%)$ & $12(15 \%)$ & $15(18 \%)$ & $13(16 \%)$ & \\
\hline pN1 & 88 & $21(24 \%)$ & $25(28 \%)$ & $12(14 \%)$ & $11(13 \%)$ & $19(22 \%)$ & \\
\hline Pancreatic invasion & 66 & $16(24 \%)$ & $22(33 \%)$ & $10(15 \%)$ & $8(12 \%)$ & $10(15 \%)$ & 0.199 \\
\hline Other loop invasion & 5 & 0 & $1(20 \%)$ & 0 & $2(40 \%)$ & $2(40 \%)$ & 0.126 \\
\hline Retroperitoneal seeding & 14 & $3(21 \%)$ & $3(21 \%)$ & $3(21 \%)$ & $3(21 \%)$ & $2(14 \%)$ & 0.726 \\
\hline Perineural invasion & 63 & $13(21 \%)$ & $20(32 \%)$ & $8(13 \%)$ & $8(13 \%)$ & $14(22 \%)$ & 0.220 \\
\hline Lymphovascular invasion & 98 & $24(25 \%)$ & $28(29 \%)$ & $10(10 \%)$ & $13(13 \%)$ & $23(24 \%)$ & 0.102 \\
\hline Suspected Lynch syndrome & 13 & $4(31 \%)$ & $2(15 \%)$ & $1(8 \%)$ & $3(23 \%)$ & $3(23 \%)$ & 0.781 \\
\hline
\end{tabular}

${ }^{\mathrm{a} C a l c u l a t e d}$ using only patients with enough available data.

${ }^{\mathrm{b}}$ Statistically significant $(P<0.05)$.

${ }^{\mathrm{C}}$ Excluded patients with pTis.

patients; 29.6\%), pancreatobiliary (45 patients; $23.8 \%$ ), gastric (26 patients; $13.8 \%)$, mixed (26 patients; $13.8 \%$ ), or null (36 patients; $19.0 \%$ ). The immunophenotypic classification was found to closely correlate with the tumor location, histological subtype, and tumor grade. Intestinal immunophenotypic adenocarcinomas were more frequently observed in the distal section (jejunum or ileum; $P=0.033)$, presenting as low-grade $(P=0.004)$ or tubular $(P=0.039)$ tumors, whereas pancreatobiliary-type carcinomas were more commonly observed in the duodenum $(P=0.033)$ and presenting as highgrade $(P=0.004)$ tumors. Undifferentiated and micropapillary carcinomas were most commonly observed in pancreatobiliary type $(P=0.039)$. Gastric-type adenocarcinomas were more frequently located in the duodenum $(P=0.033)$, but null tumors were more commonly observed in the distal sections of the intestines $(P=0.033)$. Of 13 patients with suspected Lynch syndrome, 4 had intestinal, 2 pancreatobiliary, 1 gastric, 3 mixed, and 3 null immunophenotypes of small intestinal adenocarcinomas.

\section{Survival Analysis}

Small intestinal adenocarcinoma patients with CDX2 positivity (median survival time $=71.2$ months) demonstrated significantly better survival outcomes than patients without CDX2 expression (21.5 months; $P<0.0001$; Figure 3a). However, mucin expression was found not to be associated with patient survival (Figure $3 \mathrm{~b}-\mathrm{d}$ ). When survival differences were compared with the immunophenotypic adenocarcinoma classifications of combined CDX2 and mucin expression, significant survival differences were observed between the five subgroups in our cohort $(P<0.0001$; Figure 4 a). The median survival times for small intestinal 
adenocarcinoma patients with the intestinal, pancreatobiliary, mixed, gastric, and null types were 80.8 months, 18.7 months, 26.3 months, 36.5 months, and 13.9 months, respectively. Intestinaltype adenocarcinomas demonstrated significantly better survival outcomes than all other types in our cohort $(P<0.0001$; Figure $4 \mathrm{~b})$. When pair-wise comparisons were performed, patients with intestinal-type adenocarcinomas demonstrated significantly better survival than patients with pancreatobiliary- $\quad(P<0.0001)$, gastric- $\quad(P=0.009)$, mixed- $(P=0.03)$, or null-type adenocarcinomas $(P<0.0001)$. Gastric-type adenocarcinoma patients demonstrated better survival than patients with mixed- $(P=0.04)$ or null-type tumors $(P=0.07)$. However, there were no significant survival differences between the pancreatobiliary and gastric types, pancreatobiliary and mixed types, or pancreatobiliary and null types.

\section{Association between Survival and Other Clinicopathological Factors}

Clinicopathological characteristics of the small intestinal adenocarcinomas examined in our current study, including location $(P=0.03)$, pT classification $(P=0.01)$, lymph node metastasis $(P<0.0001)$, other intestinal loop invasion $(P=0.03)$, retroperitoneal seeding $(P<0.0001)$, and lymphovascular $(P<0.0001)$ and perineural $(P=0.01)$ invasion, were also found to be significantly associated with patient survival.

\section{Multivariate Analysis of Clinicopathological Factors}

The independent prognostic significance of the immunophenotype and other clinicopathological factors, which were considered significant by univariate analysis, was further evaluated using Cox proportional hazards modeling (Table 4). According to this multivariate analysis, intestinal immunophenotype $(P=0.001)$, the absence of lymph node metastasis $(P=0.020)$, the absence of retroperitoneal seeding $(P=0.011)$, and the absence of lymphovascular invasion $(P=0.028)$ are good independent prognostic predictors of overall survival in small intestinal adenocarcinoma patients.

\section{Discussion}

To identify the clinicopathological and prognostic significance of CDX2 and mucin expression in small intestinal adenocarcinomas, we performed CDX2 and mucin protein immunohistochemical staining on a large number of patients $(n=189)$ in our present study. In these analyses, CDX2 expressing adenocarcinoma patients tended to demonstrate low-grade tumors, less lymph node metastasis, and less perineural invasion. Only a few previously reported studies have undertaken CDX2 and/or mucin protein expression analysis on small intestinal adenocarcinomas. ${ }^{11,21,34,35}$ However, due to the small number of patients who were evaluated in these earlier reports, insufficient data were available to determine the prognostic significance of the
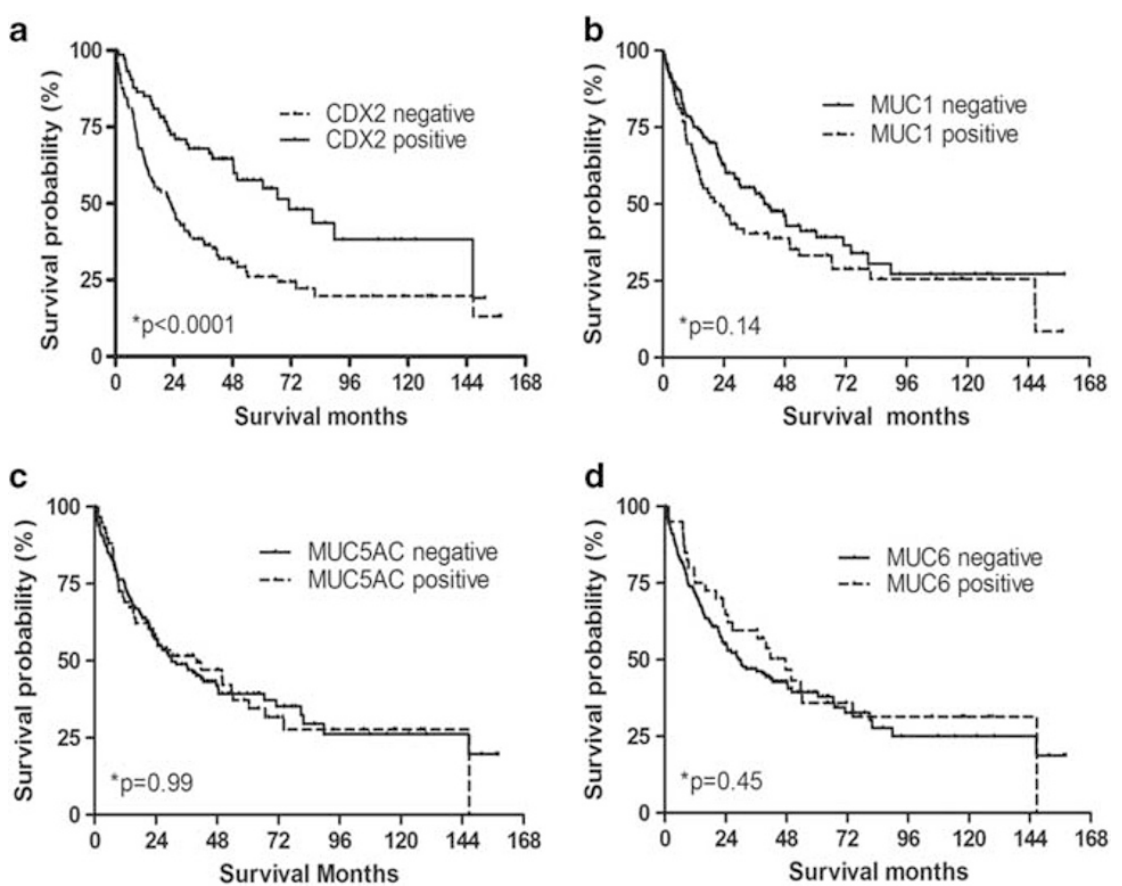

Figure 3 Expression of CDX2 and mucins with survival analysis. (a) Patients with CDX2 + adenocarcinomas demonstrated better survival rates than those with CDX2 - tumors $(P<0.0001)$. (b-d) The expression levels of (b) MUC1, (c) MUC5AC, and (d) MUC6 are not associated with patient survival. 

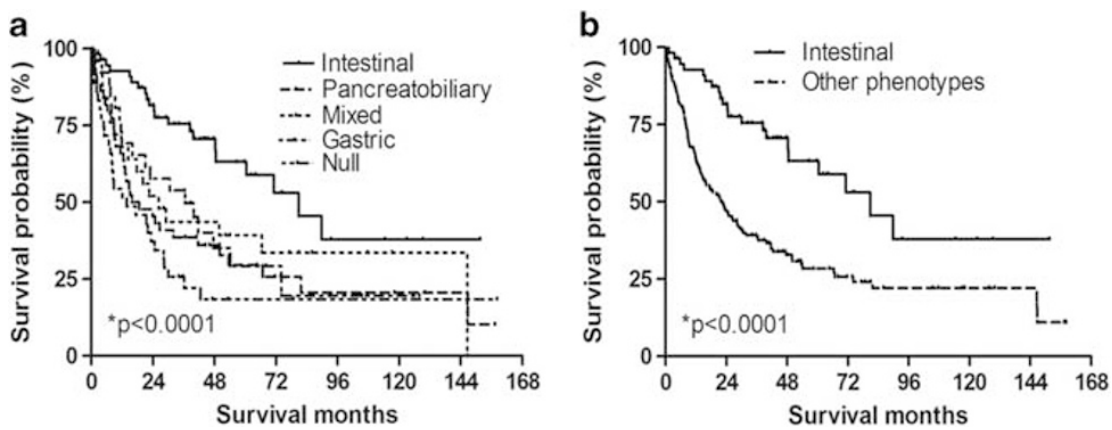

Figure 4 Small intestinal adenocarcinoma immunophenotype and survival analysis. (a) Survival differences were observed between the five small intestinal adenocarcinoma patient groups classified according to their CDX2 and mucin immunophenotype $(P<0.0001)$. (b) Patients with intestinal immunophenotype carcinomas demonstrated significantly better survival outcomes than those with non-intestinal-type tumors $(P<0.0001)$.

Table 4 Multivariate analysis of the small intestinal adenocarcinoma patients

95\% confidence interval

\begin{tabular}{|c|c|c|c|c|}
\hline Variable & Relative risk & Lower & Upper & P-value \\
\hline Intestinal immunophenotype (intestinal vs non-intestinal) & 0.424 & 0.257 & 0.699 & $0.001^{\mathrm{a}}$ \\
\hline Proximal (duodenum) vs distal (jejunum, ileum) & 1.487 & 0.946 & 2.337 & 0.086 \\
\hline pT classification & & & & 0.621 \\
\hline $\mathrm{pT} 1+\mathrm{pT} 2$ & - & - & - & 0.153 \\
\hline pT3 & 2.434 & 0.818 & 7.243 & 0.110 \\
\hline pT4 & 0.733 & 0.462 & 1.163 & 0.187 \\
\hline pN classification & 1.740 & 1.090 & 2.778 & $0.020^{\mathrm{a}}$ \\
\hline Other loop invasion & 1.432 & 0.342 & 6.002 & 0.623 \\
\hline Retroperitoneal seeding & 2.661 & 1.255 & 5.643 & $0.011^{\mathrm{a}}$ \\
\hline Perineural invasion & 1.225 & 0.781 & 1.923 & 0.377 \\
\hline Lymphovascular invasion & 1.713 & 1.059 & 2.774 & $0.028^{\mathrm{a}}$ \\
\hline
\end{tabular}

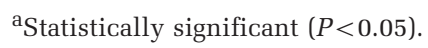

findings. Similar to CDX2 expression in other gastrointestinal cancers, including gastric and ampullary cancers, ${ }^{29,30,32,33,42}$ we found in our present study that small intestinal adenocarcinoma patients expressing CDX2 demonstrated better survival outcomes.

Small intestinal adenocarcinoma patients expressing MUC1 demonstrated nodular or infiltrative growth patterns and high-grade cancers. Previously, Zhang et $a l^{35}$ reported that poorly differentiated small intestinal adenocarcinomas more frequently express MUC1. The results of our current study concur with those of Zhang et al. Several other studies have reported that gastric cancers expressing MUC1 are associated with poorer patient survival rates. ${ }^{16,17,19}$ However, no significant survival differences were observed in our current small intestinal adenocarcinoma cohort in terms of MUC1 protein expression, which is different from the findings in gastric cancer. Lee et $a l^{11}$ have previously reported that duodenal cancers more frequently demonstrate MUC1 protein expression (10 of 14 cases; $71 \%$ ) than jejunal and ileal cancers ( 2 of 9 cases; $22 \%$ ), but they did not report any differences in terms of MUC5AC or MUC6 expression. In contrast to this previous study, we did not find any differences in MUC1 protein expression in terms of small intestinal adenocarcinoma location in our current analyses; however, we observed MUC5AC and MUC6 expression more frequently in duodenal cancers than in distal (jejunal and ileal) cancers. These differences could be due to the different sample sizes in previous studies.

Histological and immunohistochemical distinction between intestinal and pancreatobiliary cancers is well described for ampullary carcinomas. ${ }^{43}$ Intestinal adenocarcinomas consist of simple or cribriform tubular glands that are lined by tall columnar cells with pseudostratified nuclei (similar to colonic adenocarcinomas), whereas pancreatobiliary adenocarcinomas are composed of simple or branching glands that are lined by a single layer of cuboidal or columnar cells with abundant desmoplastic stroma. Similar observations have been reported for pancreatic ductal adenocarcinomas and extrahepatic cholangiocarcinomas. ${ }^{44-46}$ Immunohistochemically, intestinal ampullary tumors express various markers 
of intestinal differentiation, including CDX2 and MUC2 but not MUC1. Pancreatobiliary ampullary tumors express MUC1 but not MUC2 or CDX2. 44,45

To our knowledge, there have been no previous reports of small intestinal adenocarcinomas that were classified as intestinal or pancreatobiliary according to their morphologic and/or immunohistochemical characteristics. We here report that intestinal immunophenotypic cancers demonstrate significantly better survival outcomes than lesions of other immunophenotypes found in the small intestine. Interestingly, location of small intestinal adenocarcinoma is closely related to its immunophenotype. Duodenal cancers were more frequently gastric-type tumors, whereas distal (jejunal or ileal) tumors were more commonly intestinal-type cancers. It is plausible to explain that both stomach and proximal half of duodenum are foregut origin. Hence, the mucin protein expression patterns of duodenal cancers are similar to those of gastric cancers.

In our present study cohort, intestinal- and gastric-type tumors were more commonly low grade, whereas pancreatobiliary- and null-type cancers were typically high grade. Similar results have been noted for intraductal papillary mucinous neoplasms of the pancreas. One previous study has reported that gastric- and intestinal-type intraductal papillary mucinous neoplasms demonstrate less malignant potential than pancreatobiliary-type intraductal papillary mucinous neoplasms. ${ }^{47}$ Distler et $a l^{48}$ have reported that pancreatobiliary-type intraductal papillary mucinous neoplasm is strongly associated with advanced tumor stage in comparison with intestinal-type intraductal papillary mucinous neoplasm. Moreover, pancreatobiliary-type intraductal papillary mucinous neoplasm demonstrates higher local recurrence and metastatic rates compared with other histological subtypes of intraductal papillary mucinous neoplasm, including intestinal, gastric, and oncocytic types. In ampullary carcinoma, the pancreatobiliary type also demonstrates significantly deeper tumor invasion (that is, pT classification) and more frequent lymph nodal, perineural, duodenal, and pancreatic invasion than intestinal ampullary carcinomas. ${ }^{13,49}$ In addition, intestinal type tumors demonstrate significantly better patient survival than pancreatobiliary type neoplasms from various gastrointestinal tract tumors, including intraductal papillary mucinous neoplasm ${ }^{48}$ and ampullary ${ }^{49}$ and gastric ${ }^{50}$ carcinomas. In concordance with the results of previous studies on the prognostic implications of the morphologic and immunophenotypic classifications of other gastrointestinal tumors, the intestinal immunophenotype adenocarcinomas we examined in our present study were associated with significantly better patient survival outcomes than small intestinal adenocarcinomas of other immunophenotypes.

On the basis of our observations, non-intestinal immunophenotype small intestinal adenocarcino- mas can be used as worse prognostic indicators with lymph node metastasis, retroperitoneal seeding, and lymphovascular invasion. However, T classification was not included as a prognostic factor in this model. Because the current study is a retrospective study, further prospective studies with large number of cases are needed for solid conclusion.

In conclusion, we here investigated the expression of CDX2 and various mucins in 189 small intestinal adenocarcinoma patients. On the basis of their CDX2 and mucin expression patterns, adenocarcinomas were classified as intestinal, pancreatobiliary, gastric, mixed, or null. On the basis of the results of univariate and multivariate analyses, the patients with intestinal immunophenotype adenocarcinomas demonstrated significantly better survival outcomes than those harboring adenocarcinomas of other immunophenotypes. Hence, immunohistochemical staining for CDX2 and mucin proteins has prognostic value in surgically resected small intestinal adenocarcinoma patients.

\section{Acknowledgments}

This research was supported by the Basic Science Research Program through the National Research Foundation of Korea (2010-0004807), which is funded by the Ministry of Education, Science, and Technology, and also by a grant (2013-554) from the Asan Institute for Life Sciences, Seoul, Korea. This work was presented, in part, at the 24th annual meeting of the European congress of pathology, Prague, Czech Republic, September 8-12th, 2012.

\section{Disclosure/conflict of interest}

The authors declare no conflict of interest.

\section{References}

1 Siegel R, Naishadham D, Jemal A. Cancer statistics, 2013. CA Cancer J Clin 2013;63:11-30.

2 Chang HK, Yu E, Kim J, et al. Adenocarcinoma of the small intestine: a multi-institutional study of 197 surgically resected cases. Hum Pathol 2010;41: 1087-1096.

3 Arber N, Hibshoosh H, Yasui W, et al. Abnormalities in the expression of cell cycle-related proteins in tumors of the small bowel. Cancer Epidemiol Biomarkers Prev 1999;8:1101-1105.

4 Blaker $\mathrm{H}$, Helmchen B, Bonisch A, et al. Mutational activation of the RAS-RAF-MAPK and the Wnt pathway in small intestinal adenocarcinomas. Scand J Gastroenterol 2004;39:748-753.

5 Sutter T, Arber N, Moss SF, et al. Frequent K-ras mutations in small bowel adenocarcinomas. Dig Dis Sci 1996;41:115-118.

6 Wheeler JM, Warren BF, Mortensen NJ, et al. An insight into the genetic pathway of adenocarcinoma of the small intestine. Gut 2002;50:218-223. 
7 Lee HJ, Lee OJ, Jang KT, et al. Combined loss of E-cadherin and aberrant beta-catenin protein expression correlates with a poor prognosis for small intestinal adenocarcinomas. Am J Clin Pathol 2013;139: 167-176.

8 Hollingsworth MA, Swanson BJ. Mucins in cancer: protection and control of the cell surface. Nat Rev Cancer 2004;4:45-60.

9 Zheng H, Takahashi H, Nakajima T, et al. MUC6 downregulation correlates with gastric carcinoma progression and a poor prognosis: an immunohistochemical study with tissue microarrays. J Cancer Res Clin Oncol 2006;132:817-823.

10 Cozzi PJ, Wang J, Delprado W, et al. MUC1, MUC2, MUC4, MUC5AC and MUC6 expression in the progression of prostate cancer. Clin Exp Metastasis $2005 ; 22: 565-573$.

11 Lee MJ, Lee HS, Kim WH, et al. Expression of mucins and cytokeratins in primary carcinomas of the digestive system. Mod Pathol 2003;16:403-410.

12 Dekker JRJ, Büller HA, Einerhand AWC. The MUC family: an obituary. Trends Biochem Sci 2002;27: 126-131.

13 Kawabata Y, Tanaka T, Nishisaka T, et al. Cytokeratin 20 (CK20) and apomucin 1 (MUC1) expression in ampullary carcinoma: correlation with tumor progression and prognosis. Diagn Pathol 2010;5:75.

14 Aloysius MM, Zaitoun AM, Awad S, et al. Mucins and CD56 as markers of tumour invasion and prognosis in periampullary cancer. Br J Surg 2010;97:1269-1278.

15 Ilhan O, Han U, Onal B, et al. Prognostic significance of MUC1, MUC2 and MUC5AC expressions in gastric carcinoma. Turk J Gastroenterol 2010;21:345-352.

$16 \mathrm{Li}$ XH, Zheng HC, Wang ZG, et al. The clinicopathological and prognostic significance of MUC-1 expression in Japanese gastric carcinomas: an immunohistochemical study of tissue microarrays. Anticancer Res 2008;28:1061-1067.

17 Kocer B, Soran A, Kiyak G, et al. Prognostic significance of mucin expression in gastric carcinoma. Dig Dis Sci 2004;49:954-964.

18 Ajisaka H, Fushida S, Yonemura Y, et al. Expression of insulin-like growth factor-2, c-MET, matrix metalloproteinase-7 and MUC-1 in primary lesions and lymph node metastatic lesions of gastric cancer. Hepatogastroenterology 2001;48:1788-1792.

19 Utsunomiya $\mathrm{T}$, Yonezawa $\mathrm{S}$, Sakamoto $\mathrm{H}$, et al. Expression of MUC1 and MUC2 mucins in gastric carcinomas: its relationship with the prognosis of the patients. Clin Cancer Res 1998;4:2605-2614.

20 Silberg DG, Swain GP, Suh ER, et al. Cdx1 and cdx2 expression during intestinal development. Gastroenterology 2000;119:961-971.

21 Werling RW, Yaziji H, Bacchi CE, et al. CDX2, a highly sensitive and specific marker of adenocarcinomas of intestinal origin: an immunohistochemical survey of 476 primary and metastatic carcinomas. Am J Surg Pathol 2003;27:303-310.

22 Freund JN, Domon-Dell C, Kedinger M, et al. The Cdx1 and Cdx-2 homeobox genes in the intestine. Biochem Cell Biol 1998;76:957-969.

23 Mallo GV, Soubeyran P, Lissitzky JC, et al. Expression of the Cdx1 and Cdx2 homeotic genes leads to reduced malignancy in colon cancer-derived cells. J Biol Chem 1998;273:14030-14036.

24 Roessler K, Monig SP, Schneider PM, et al. Coexpression of CDX2 and MUC2 in gastric carcinomas: correlations with clinico-pathological parameters and prognosis. World J Gastroenterol 2005;11:3182-3188.

25 Ha Kim G, Am Song G, Youn Park D, et al. CDX2 expression is increased in gastric cancers with less invasiveness and intestinal mucin phenotype. Scand J Gastroenterol 2006;41:880-886.

26 Uozaki H, Barua RR, Minhua S, et al. Transcriptional factor typing with SOX2, HNF4aP1, and CDX2 closely relates to tumor invasion and Epstein-Barr virus status in gastric cancer. Int J Clin Exp Pathol 2011;4:230-240.

27 Park DY, Srivastava A, Kim GH, et al. CDX2 expression in the intestinal-type gastric epithelial neoplasia: frequency and significance. Mod Pathol 2010;23: 54-61.

28 Okayama H, Kumamoto K, Saitou K, et al. CD44v6, MMP-7 and nuclear Cdx2 are significant biomarkers for prediction of lymph node metastasis in primary gastric cancer. Oncol Rep 2009;22:745-755.

29 Ge J, Chen Z, Wu S, et al. A clinicopathological study on the expression of cadherin-17 and caudal-related homeobox transcription factor (CDX2) in human gastric carcinoma. Clin Oncol (R Coll Radiol) 2008;20: 275-283.

30 Fan Z, Li J, Dong B, et al. Expression of Cdx2 and hepatocyte antigen in gastric carcinoma: correlation with histologic type and implications for prognosis. Clin Cancer Res 2005;11:6162-6170.

31 Bai Z, Ye Y, Chen D, et al. Homeoprotein Cdx2 and nuclear PTEN expression profiles are related to gastric cancer prognosis. APMIS 2007;115:1383-1390.

32 Mizoshita T, Tsukamoto T, Nakanishi H, et al. Expression of Cdx2 and the phenotype of advanced gastric cancers: relationship with prognosis. J Cancer Res Clin Oncol 2003;129:727-734.

33 Hansel DE, Maitra A, Lin JW, et al. Expression of the caudal-type homeodomain transcription factors CDX $1 / 2$ and outcome in carcinomas of the ampulla of Vater. J Clin Oncol 2005;23:1811-1818.

34 Overman MJ, Pozadzides J, Kopetz S, et al. Immunophenotype and molecular characterisation of adenocarcinoma of the small intestine. Br J Cancer 2010;102: 144-150.

35 Zhang MQ, Lin F, Hui $\mathrm{P}$, et al. Expression of mucins, SIMA, villin, and CDX2 in small-intestinal adenocarcinoma. Am J Clin Pathol 2007;128:808-816.

36 Chu PG, Schwarz RE, Lau SK, et al. Immunohistochemical staining in the diagnosis of pancreatobiliary and ampulla of Vater adenocarcinoma: application of CDX2, CK17, MUC1, and MUC2. Am J Surg Pathol 2005;29:359-367.

37 Kaimaktchiev V, Terracciano L, Tornillo L, et al. The homeobox intestinal differentiation factor CDX2 is selectively expressed in gastrointestinal adenocarcinomas. Mod Pathol 2004;17:1392-1399.

38 Moskaluk CA, Zhang H, Powell SM, et al. Cdx2 protein expression in normal and malignant human tissues: an immunohistochemical survey using tissue microarrays. Mod Pathol 2003;16:913-919.

39 Hamiton SR, Nakamura S-I, Bosman FT, et al. Carcinoma of the colon and rectum, In: Bosman FT, Carneiro F, Hruban RH, Theise ND (eds). WHO Classification of Tumours of the Digestive System, 4th edn. IARC: Lyon, France; 2010, pp 134-146.

40 Kim G, Chung JY, Jun SY, et al. Loss of S100A14 expression is associated with the progression of adenocarcinomas of the small intestine. Pathobiology 2013;80:95-101. 
41 Peltomaki P, Offerhaus GJA. Vasen HFA. Lynch syndrome, In: Bosman FT, Carneiro F, Hruban RH, Theise ND (eds) WHO Classification of Tumours of the Digestive System, 4th edn. IARC: Lyon, France; 2010, pp 152-155.

42 Bai YQ, Yamamoto H, Akiyama Y, et al. Ectopic expression of homeodomain protein CDX2 in intestinal metaplasia and carcinomas of the stomach. Cancer Lett 2002;176:47-55.

43 Kimura W, Futakawa N, Yamagata S, et al. Different clinicopathologic findings in two histologic types of carcinoma of papilla of Vater. Jpn J Cancer Res 1994;85:161-166.

44 Klimstra DS, Albores-Saavedra J, Hruban RH, et al. Adenomas and other premalignant neoplastic lesions, In: Bosman FT, Carneiro F, Hruban RH, Theise ND (eds). WHO Classification of Tumours of the Digestive System, 4th edn. IARC: Lyon, France; 2010, pp 83-86.

45 Albores-Saavedra J, Hruban RH, Klimstra DS, et al. Invasive adenocarcinoma of the ampullary region, In: Bosman FT, Carneiro F, Hruban RH, Theise ND (eds).
WHO Classification of Tumours of the Digestive System, 4th edn. IARC: Lyon, France; 2010, pp 87-91.

46 Shepherd NA, Carr NJ, Howe JR, et al. Carcinoma of the small intestine, In: Bosman FT, Carneiro F, Hruban RH, Theise ND (eds) WHO Classification of Tumours of the Digestive System, 4th edn. IARC: Lyon, France; 2010, pp 98-101.

47 Takasu N, Kimura W, Moriya T, et al. Intraductal papillary-mucinous neoplasms of the gastric and intestinal types may have less malignant potential than the pancreatobiliary type. Pancreas 2010;39:604-610.

48 Distler $\mathrm{M}$, Kersting S, Niedergethmann $\mathrm{M}$, et al. Pathohistological subtype predicts survival in patients with intraductal papillary mucinous neoplasm (IPMN) of the pancreas. Ann Surg 2013;258:324-330.

49 Moriya T, Kimura W, Hirai I, et al. Expression of MUC1 and MUC2 in ampullary cancer. Int J Surg Pathol 2011;19:441-447.

50 Solcia E, Klersy C, Vanoli A, et al. The contribution of cell phenotype to the behavior of gastric cancer. Gastric Cancer 2013;16:462-471. 